\title{
Dysexecutive syndrome in schizophrenia: A cross-cultural comparison between Japanese and British patients
}

\author{
H. Ihara ${ }^{\mathrm{a}, *}$, G.E. Berrios ${ }^{\mathrm{a}}$ and P.J. McKenna ${ }^{\mathrm{b}}$ \\ ${ }^{a}$ Department of Psychiatry, University of Cambridge, \\ Hills Road, Cambridge, UK \\ ${ }^{\mathrm{b}}$ Cambridge Psychiatric Rehabilitation Service, \\ Fulbourn Hospital, Cambridge, UK
}

\begin{abstract}
This study tested the hypothesis that the dysexecutive syndrome of schizophrenia is impervious to cultural differences. 18 Japanese and 22 British patients and 14 Japanese and 19 British control subjects were compared on the BADS (Behavioural Assessment of Dysexecutive Syndrome), a comprehensive neuropsychological assessment battery, designed for 'ecological validity', and other measures of frontal executive functions (EFs). Both cultural groups of schizophrenic patients showed equally severe impairment in most executive tests including the BADS (verbal fluency and intelligence were less impaired). Irrespective of cultural origin, similar neuropsychological deficits were found in patients with minimal intellectual deterioration. Our study suggests that socio-cultural background does not affect the dysexecutive profile of schizophrenia very much, and that neuropsychological assessment may possibly provide added clinical information relevant to the management and rehabilitation of schizophrenic patients across different cultures.
\end{abstract}

Keywords: Cognitive dysfunction, dysexecutive syndrome, cross-cultural study, Japanese

\section{Introduction}

There has been less empirical research to ascertain the influence of cultural context on the cognitive disorders that accompany schizophrenia than there has been into its influence on the rest of schizophrenic symp-

* Corresponding author / Present address: H. Ihara, National South Hanamaki Hospital, Suwa 500, Hanamaki, Iwate, Japan 025-0033. Tel.: +81 19824 0511; Fax: +81 19824 1721; E-mail: mhi202@ shanamaki.hosp.go.jp. tomatology. Indeed, work in the latter area started early $[28,40]$ and the view is still accepted that the symptoms, course and outcome of schizophrenia are less severe in non-western countries or non-Caucasian ethnic groups $[6,8,32]$. WHO studies [58] have also found that culture seems to influence the profile of positive symptoms such as delusion of control, persecutory delusion, characteristic auditory hallucinations, and distortions of self-perception. It has also been reported that Japanese subjects suffering from schizophrenia are more self-contained, reclusive, emotionally shallow, and socially withdrawn compared with non-Japanese patients [16,24,49].

However, as Murphy [39] has pointed out, such symptoms may not be relevant to the illness process, and hence their nature and duration are unlikely to matter much from a neurobiological viewpoint. This is because such highly noticeable characteristics yield to less marked but persistent developments of pervasive deterioration in distinguishing schizophrenia from other psychoses. After exacerbation and remission of delusions and hallucinations, a considerable proportion of patients fall into a state of severe deterioration with prominent cognitive deficits such as lack of spontaneity, blunted affect, marked lethargy, loss of forward planning and difficulty with abstract thinking.

These cognitive deficits are more or less synonymous with what, in neuropsychology, has recently been called the 'dysexecutive syndrome' (DES) [3]. This syndrome refers to the impairment of executive functions (EFs) whose putative anatomical substrata are the frontal lobes and basal ganglia. Over the past decade, EFs have become an important field of research in schizophrenia [4,18-21,31,33,43,50,51]. It is claimed that the dysfunction of EFs in schizophrenia is disproportionate to the overall level of the other domains of higher brain function $[10,15,17,27,56]$. To clinicians working in rehabilitation settings, these symptoms are of primary concern, as they impede recovery. An un- 
resolved question is whether culture modifies dysexecutive patterns. If it did not, the view that schizophrenia is the same neurobiological disorder across cultures and that DES may be its basic psychopathology will be reinforced.

The comparison of Japan with Britain is germane for the following reasons. Firstly, the two cultures have different historical traditions and national traits. Japan remains under the influence of a Confucianism characterised by family-centricity, socio-centricity and collectivism. Britain is an example of a Western society that emphasises individualism, independence and selfreliance. In this regard, differences in thematic content for positive symptoms between Japanese and Caucasian schizophrenic patients have been reported [16, 24,49]. Secondly, the Japanese language differs from the most Indo-European languages in the following respect: there are two character systems that comprise the Japanese language, one of them consisting of a large number of complex pictorial characters. Due to the possibility that such complex pictorial characters are processed in a different manner from that associated with phonemic characters, long-term exposure to this cognitive-linguistic environment may produce some sorts of neuropsychological variations, such as greater relative right hemisphere activation [22,37]. Thirdly, both countries are industrialised, urbanised and literate, so that potentially confounding factors such as differences in education, intelligence and verbal capacity may be less of a problem.

This study was designed to investigate the extent and variation of the dysexecutive syndrome in both cultural groups. Its aim is to clarify whether patients from both cultures perform differently on tests of verbal fluency, mental-set shifting, abstract concept formation, goaldirectedness and planning and whether level of intelligence is a factor in their performance (e.g. [10,51]).

\section{Methods}

\subsection{Participants}

A total of 73 subjects participated in this study: 18 Japanese schizophrenic patients; 22 British schizophrenic patients; 14 Japanese and 19 British healthy volunteer comparison subjects.

The Japanese patients with schizophrenia who participated in this study were recruited from Muroi Hospital, Oyama Fujimidai Hospital and Nasu Hillside Hospital, all of which lie in Tochigi Prefecture, Japan, ap- proximately $100 \mathrm{~km}$ north of Tokyo. All the Japanese patients were being treated with neuroleptics at the time of the neuropsychological assessment.

The British patients with schizophrenia who took part in the study were recruited from a psychiatric rehabilitation service in Cambridge. They were all Caucasians and native English speakers. Their scores on the NART [42] and the duration and type of their education indicated that they were considered to be of average premorbid intelligence. All were taking neuroleptics.

Semi-structured diagnostic interviews revealed that all Japanese and British patients met the criteria for schizophrenia according to the Diagnostic and Statistical Manual of Mental Disorders: Fourth Edition (DSMIV) [2] and the International Classification of Diseases (ICD-10) [59]. Patients with any co-morbidity of other mental illnesses or a history of electroconvulsive therapy within the last two years were excluded. For those who were taking anticholinergic agents, doses were below the level affecting memory test performance in schizophrenia, i.e. the range $5-15 \mathrm{mg}$ procyclidine daily [52]. Symptomatological evaluations for the schizophrenic groups were made according to semistructured clinical interviews and standardised rating scales for the Positive and Negative Syndrome Scale (PANSS) [25] by an English speaking Japanese psychiatrist (H.I.) and using the patients' case records. We found no difference in symptomatology rated by the PANSS and the neuroleptic dose of chlorpromazine equivalents $[11,26]$ in the two groups with schizophrenia (Table 2).

Nineteen British control subjects responded to advertisements for paid volunteers at the Medical Research Council Cognition and Brain Sciences Unit, Cambridge and co-operated in obtaining norms of each neuropsychological test. The British patients with schizophrenia and the British control subjects were matched for duration of education and NART-based estimated premorbid intelligence. Fourteen Japanese control subjects, including housewives, cleaners, farmers and students, were selected to match the other groups for age. Most of them were paid volunteers recruited by advertisement in the local community. We found no significant statistical age differences among the four groups $(F=2.07, d f=3,69, p=0.112)$. All subjects were screened for a history of head injury followed by loss of consciousness, substance abuse and general somatic conditions that might affect neuropsychological performance. We excluded those who had been previously diagnosed as learning disorders and met the criteria of mental retardation and learning disorders, according to 
Table 1

Demographic and baseline variables of the Japanese schizophrenic patients, the British schizophrenic patients, and the Japanese and British healthy control subjects (means and, in parenthesis, standard deviations for age, duration of illness, duration of education, and NART-IQ)

\begin{tabular}{lllll}
\hline & $\begin{array}{l}\text { Japanese Schizophrenic } \\
\text { Patients }\end{array}$ & $\begin{array}{l}\text { British Schizophrenic } \\
\text { Patients }\end{array}$ & $\begin{array}{l}\text { Japanese Control } \\
\text { Subjects }\end{array}$ & $\begin{array}{l}\text { British Control } \\
\text { Subjects }\end{array}$ \\
\hline $\mathrm{N}$ & 18 & 22 & 14 & 19 \\
Female: male & $10: 8$ & $7: 15$ & $9: 5$ & $17: 2$ \\
Age & $44.2(10.6)$ & $37.0(9.5)$ & $38.4(12.3)$ & $43.4(11.2)$ \\
Duration of illness & $17.8(10.6)$ & $14.1(8.9)$ & & \\
Inpatients: outpatients & $17: 1$ & $11: 11$ & \\
Duration of education & $12.3(2.5)$ & $12.0(2.2)$ & & $103.2(6.1)$ \\
NART-IQ & & $104.4(13.8)$ & & \\
\hline
\end{tabular}

Note.NART $=$ National Adult Reading Test [42].

Table 2

The symptoms rated by Positive and Negative Syndrome Scale [25] and the neuroleptic dose of chlorpromazine equivalents in the Japanese and British schizophrenic patients. (means and, in parenthesis, standard deviations)

\begin{tabular}{lcc}
\hline & Japanese Schizophrenic patients & British Schizophrenic patients \\
\hline Positive syndrome & $15.5(5.8)$ & $17.1(5.2)$ \\
Negative syndrome & $20.7(4.5)$ & $22.8(6.3)$ \\
Composite index & $-5.2(6.1)$ & $-5.7(6.5)$ \\
General psychopathology & $34.3(10.3)$ & $41.1(10.0)$ \\
Anergia & $9.6(2.7)$ & $11.4(3.7)$ \\
Thought disturbance & $9.6(4.0)$ & $10.3(3.4)$ \\
Activation & $6.4(2.4)$ & $7.6(2.8)$ \\
Paranoid/belligerence & $5.4(2.1)$ & $6.5(1.9)$ \\
Depression & $6.9(2.5)$ & $8.2(3.1)$ \\
Neuroleptics (= Chlorpromazine) (mg/day) & $625.0(535.6)$ & $608.1(473.5)$ \\
\hline
\end{tabular}

DSM-IV, at the time of the neuropsychological assessment.

In order to test the DES hypothesis of schizophrenia, the possibility that the subject's poor performance on executive tests merely reflects low intellectual functioning must be discounted. For this purpose, we matched the four samples for the means of either pre-morbid or current intelligence. For the British schizophrenic sample, we excluded subjects whose mother tongue was not English, who could not perform the tests, who were either uncooperative or emotionally unstable, and those whose National Adult Reading Test (NART) [42] IQ was $<84$. The British control sample (normals) was selected to match the British schizophrenic patients for the means of duration of education and NART-based premorbid IQ. Japanese schizophrenic patients were also selected to match the British schizophrenic sample on the basis of equivalent average scores on the Raven Advanced Progressive Matrices (RAPM) [46], and also duration of education and duration of illness. The RAPM seems less susceptible to cultural factors than other tests such as the Wechsler Adult Intelligent Scale [55] and NART [23]. We also deliberately matched British and Japanese control subjects on the basis of RAPM-base current intelligence when recruiting the latter control subjects.
Table 1 provides demographic and baseline variables of the Japanese schizophrenic patients, British schizophrenic patients, and healthy control subjects.

We obtained a written form of consent from all of the participants after the procedure had been fully explained. We also obtained ethical approval from both the Cambridge Local Research Ethics Committee and the Clinical Management Committee of each Japanese hospital.

\subsection{Methods}

The extended battery of neuropsychological tests of EFs applied to all three groups included: Verbal Fluency Test (VFT) [35]; Trail Making Test (TMT) [47]; Modified Wisconsin Card Sorting Test (MCST) [41]; Stroop Colour Word Test (SCW) [53]; Goal Neglect Test (GNT) [14]; Behavioural Assessment of Dysexecutive Syndrome (BADS) [57]. General details of the GNT and the BADS are provided here. In terms of the other tests, we have given a brief account of their application to Japanese subjects, since a full explanation would be unnecessary due to their clinical popularity. More detailed descriptions can be obtained from the literature shown above. Table 3 shows cognitive domains assessed by the neuropsychological test battery. 
Table 3

Cognitive domains examined by the neuropsychological test battery

\begin{tabular}{ll}
\hline Cognitive Domain & Neuropsychological Tests \\
\hline Intelligence & Estimated premorbid intelligence \\
NART & Current intelligence, fluid intelligence \\
RAPM & \\
Formal Executive Functions & Initiation and fluency of speech \\
VFT & Visuo-motor tracking and mental 'set-shifting \\
TMT: B-A & Abstract concept formation \\
MCST & Inhibitory control of habitual behaviour \\
SCW & Retention of goal-directed mental-set \\
GNT & \\
Everyday Executive Functions & \\
Rule Shift Cards & Inhibition of pre-learned behaviour \\
Action Programme & Development of an action plan \\
Key Search & Strategy-forming \\
Temporal Judgement & Stepwise temporal estimation \\
Zoo Map & Forward planning \\
Modified Six Elements & Planning and organisation of behaviour \\
BADS Total Score & Everyday executive functions \\
\hline Note. NART = National Adult Reading Test [42]; RAPM = Raven Advanced \\
Progressive Matrices [46]; VFT = Verbal Fluency Test [36]; TMT = Trail Making \\
Test [47]; MCST = Modified Card Sorting Test [41]; SCW = Stroop Colour Word \\
Test [53]; GNT = Goal Neglect Test [14]; BADS = Behavioural Assessment of \\
Dysexecutive Syndrome [57]. &
\end{tabular}

\subsubsection{Verbal Fluency Test (VFT)}

This test asks subjects to produce orally as many words as possible beginning with a particular letter in sixty seconds. In this project, the initial letters ' $F$ ', 'A' and ' $\mathrm{S}$ ' are used for British subjects, whereas letters ' $\mathrm{H}$ ', 'A' and ' $\mathrm{S}$ ' are applied to Japanese individuals. The rationale for comparison between English VFT and its Japanese equivalent is provided by the following: the total number of words starting with the letter ' $F$ ', 'A' or ' $\mathrm{S}$ ' comprise $23.1 \%$ of the entire English vocabulary (F: 4.9\%; A: 5.3\%; S: 12.9\%) (Oxford English Dictionary), whereas the total number of words beginning with the letter ' $H$ ', 'A' or ' $S$ ' account for $26.3 \%$ of the whole Japanese vocabulary (H: $9.8 \%$; A: 3.2\%; S: $13.3 \%$ ) (Shinchosha Modern Japanese Dictionary). We used the total number of all correct words, produced by subjects, as their score.

\subsubsection{Trail Making Test (TMT)}

All subjects performed both Parts A and B of the TMT. For Japanese subjects, Hiragana characters are used in Part B, according to the dictionary arrangement of the Japanese syllabary. Each Japanese character is placed on the corresponding letter from the Roman alphabet in the correct order. For example, the first Hiragana character 'あ' (pronunciation: short ' $\mathrm{a}$ ') is placed exactly in the same place as 'A', and the second letter ' $い$ ' (pronunciation: short ' $i$ ') in the same place as ' $\mathrm{B}$ '. The time taken for Part B subtracted from the time taken for Part A was scored. A standardised score based on control means and standardised deviation is multiplied by ' -1 ' in order to equalise the valence of standardised scores on the TMT with the other tests: the lower the score, the greater the impairment.

\subsubsection{Modified Wisconsin Card Sorting Test (MCST)}

This study applied Nelson's modified version [41], in which the subject is given clear information about the change in a rule at the time of its occurrence. The number of categories subjects attained was recorded. We applied the same procedure to both Japanese and British participants. Instructions were given to Japanese subjects in Japanese.

\subsubsection{The Stroop Colour Word Test (SCW)}

This consists of two tasks, in both of which subjects are given a page with a series of 112 colour words printed in different colours of ink. The number of correct responses in the second task (Colour Word Task) is scored. A Japanese version with the same number of colour words printed in different colours was administered to Japanese subjects. The colour words in the Japanese versions were similar in number of syllables to their English counterparts.

\subsubsection{Goal Neglect Test (GNT)}

In this test, a series of capital letters or numbers is presented in the middle of a computer screen. Each trial consists of 13 stimulus pairs (either letter or number 
pairs) and begins with the word READY, which is replaced by the instruction WATCH RIGHT or WATCH LEFT when the experimenter presses a key. A stimulus sequence then begins, composed of the first 10 pairs and one + or - cue followed by the second three pairs. Subjects are given the task of naming the letters on the side indicated by the first instruction, for the first ten pairs, and those indicated by the second side instruction (SSI), according to the rule that the + symbol means 'watch right' and - 'watch left', for the last three pairs. Subjects make a block of twelve experimental trials consisting of six 'stay' trials (WATCH RIGHT followed by + and WATCH LEFT followed by -) and six 'switch' trials (WATCH RIGHT followed by - and WATCH LEFT followed by + ). The test is performed after a few practice trials. Whether or not subjects responds appropriately to the SSI is assessed. Japanese subjects performed a Japanese version with Hiragana characters, instead of letters of the Roman alphabet.

\subsubsection{Behavioural Assessment of Dysexecutive Syndrome (BADS)}

This consists of six sub-tests. From each a 'summary profile score' is obtained (with a maximum of 4 and minimum of 0 ) and these are added up to produce an overall score (out of 24). Compared with traditional tests of EFs, this new battery has the advantage of 'ecological validity', i.e. its tasks are reflective of everyday cognitive contexts. We applied the same procedure to both Japanese and British participants, although instruction to Japanese subjects was spoken in Japanese. We provide a brief explanation of the tests here, while the test manual shows more detailed descriptions.

\subsubsection{Rule shift cards}

This test consists of 'Trial 1' and '2', both of which use the same set of 21 spiral-bound non-court playing cards, including both black and red cards which appear at random. Its purpose is to assess subjects' ability to perform a given task procedure appropriately, to shift from one rule to another, and to inhibit a pattern of response learned immediately before the task is performed. In Trial 1 they are asked to say 'Yes' to a red card and 'No' to a black card, as cards are turned over one by one. In Trial 2 they are asked to say 'Yes' if the card is the same colour as the previous one, otherwise to say No'.

\subsubsection{Action programme}

This test aims to examine the development of an action plan by the subject. They are given a rectangular stand into which a large plastic beaker and a thin tube are placed. The beaker is two thirds full of water and covered by a removable lid with a small central hole in it. The tube includes a small piece of cork at the bottom. Both the beaker and the tube are transparent. Subjects are asked to get the cork out of the tube, using any of the following materials: a metal, L-shaped rod which is too short to reach the cork; a small screw-top container; and its unscrewed top. A stipulation is that subjects are forbidden to lift up the stand, the tube or the beaker or to touch the lid with their fingers. Subjects are required to identify and organise the following procedures: to remove the lid from the beaker, using the wire; to attach the top to the small container and use this to take water from the beaker and pour it into the tube in order to float the cork to the top.

\subsubsection{Key search}

Subjects are given a piece of paper in whose centre a $10 \mathrm{~cm}$ square is drawn with a small dot five $\mathrm{cm}$ below it. They are asked to imagine that this square is a large field and that somewhere in this field they have lost their keys. The black dot denotes their starting point. Their task is to draw a line to show how the field can be searched so that the keys will be found.

\subsubsection{Temporal judgement}

This test includes four questions which require subjects to estimate the length of time required for certain activities. They are told that they are not expected to answer these questions precisely but that they should make a sensible guess. The questions are: "How long does it take to have a routine dental check up?"; "How long does it take for a window cleaner to clean the windows of an average size house?"; "How long do most dogs live for?"; and "How long does it take to blow up a party balloon? ".

\subsubsection{Zoo map}

This test consists of two parts, both of which ask subjects to show how they would visit designated places on a map of a zoo without breaking the rule of using designated paths once only. There are only four variations on a route which conform to the rules. The first part of the test requires subjects to plan the order in which to visit the locations. The second part gives them explicit instructions about how to plan a route in order to avoid errors. Japanese subjects were given Japanese Zoo Maps in which all place names and relevant instructions were written in Japanese. 


\subsubsection{Modified six elements test}

Subjects are set three tasks - dictation, arithmetic and picture naming - each of which consists of Parts $\mathrm{A}$ and $\mathrm{B}$, hence six sub-tasks in total. They must obey two rules. First, they have to attempt at least one thing from each of the six sub-tasks within ten minutes. That is, they must at least attempt each of the six sub-tasks. Second, they must not do two parts of the same task, one after the other. Japanese subjects were given a Japanese instruction sheet in which all instructions were written in Japanese.

\subsection{Analyses}

We used the SPSS V8 Package for descriptive and inferential statistical analysis. Analysis of covariance (ANCOVA), with adjustment for the effects of the RAPM-based general intelligence, was used to study both the main effects of condition (schizophrenic patients versus control subjects) and nationality (Japanese versus British), as well as their interactions, on raw scores of neuropsychological tests.

Where homogeneity of variance differed significantly between groups according to the Levene test, additional non-parametric analyses (Kruskal-Wallis tests) were performed on the variables. In fact, the Levene test revealed significant differences of homogeneity of variance between groups on some of the measures, such as the TMT, the SCW, the GNT and some sub-tests of the BADS. But non-parametric analyses did not alter the main findings of these tests.

In Fig. 1, raw test scores were transformed to $\mathrm{Z}$ scores based on the means and standard deviations of the British normal comparison group. The GNT was excluded from the analysis as its scores showed a bimodal rather than normal distribution [13]. Where high scores indicated poorer performance, scores were transformed (direction reversed) so that high scores always denoted better cognitive performance.

\section{Results}

RAPM scores were submitted to a preliminary analysis of variance (ANOVA) using two between-subject factors: clinical condition (schizophrenic patients versus control subjects) and nationality (Japanese and British). The ANOVA revealed a significant main effect of condition $(F=30.746, d f=3,68, p<0.001)$. ANCOVA, which controlled for the RAPM-based current intelligence, revealed a significant main effect of clinical condition on neuropsychological performance. Schizophrenic patients performed worse on most conventional executive tests, including the TMT $(F=6.213, d f=1,68, p=0.015)$, the MCST $(F=7.482, d f=1,60, p=0.008)$, and the SCW $(F=8.780, d f=1,66, p=0.004)$. A newly created computerised letter-monitoring test, the GNT, also revealed a significant inter-group difference in terms of clinical condition $(F=10.434, d f=$ $1,54, p=0.002)$. At the same time, a difference between schizophrenic patients and control subjects on the VFT did not reach the level of statistical significance $(F=0.008, d f=1,68, p=0.930)$. Schizophrenic patients also scored worse on three sub-tests and the profile score of the newly-devised comprehensive neuropsychological battery of EFs: the Rule Shift Cards $(F=4.115, d f=1,65, p=0.047)$; the Zoo Map $(F=9.390, d f=1,66, p=0.003)$; Modified Six Elements $(F=8.955, d f=1,65, p=0.004)$; and the Profile score $(F=14.225, d f=1,65, p<0.001)$. Nationality as a main effect was not significant for any cognitive measures, except the GNT. The interaction of condition by nationality was not significant for any cognitive measures.

We further examined the relationship between EFs and general intellectual function by separately analysing the performance of patients with minimal IQ decline. Based on Raven's conversion table [45], RAPM raw scores for each schizophrenic subject were converted to normalised deviation IQ. Ten British patients with a discrepancy of less than 15 points between NART estimated IQ and the RAPM current IQ were chosen. Basic variables in this group were as follows (means and, in parentheses, standard deviations): education 12.2 (2.7); NART IQ 99.5 (12.0); RAPM IQ 104.0 (12.4). Seven Japanese schizophrenic patients with RAPM IQ equal to or higher than 90 were also chosen. Basic variables in this group were as follows: education 12.5 (2.8); RAPM IQ 99.2 (13.2). As a test analogous to the NART does not exist in the Japanese language, it was not possible to estimate exactly premorbid intelligence in the Japanese patients. They were chosen, however, as the most intelligent amongst the 18 Japanese patients on the basis of current IQs as indicated by RAPM scores. These two groups were, finally, compared with the two control groups in terms of neuropsychological scores, using analysis of variance (ANOVA) to study both the main effects of condition and nationality, as well as their interactions, on raw scores of neuropsychological tests. But, none of these re-computations changed the results very much (Table 5). 
Table 4

ANCOVA results upon neuropsychological results, using the RAPM score, as covariate, and two between-subject factors: condition (schizophrenic patients versus control subjects) and nationality (Japanese versus British)

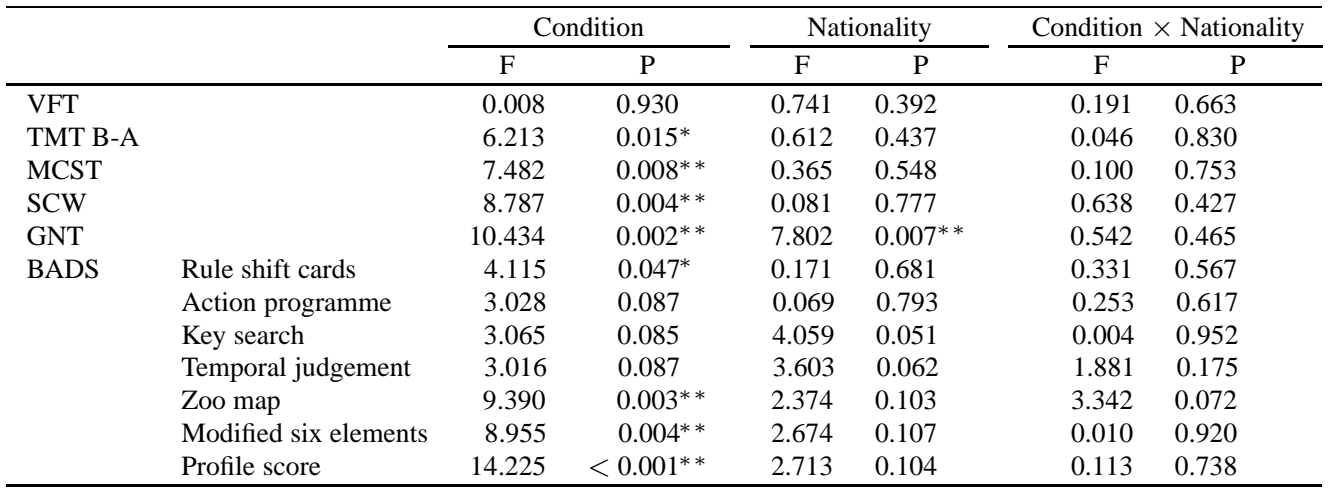

${ }^{*} p<0.05 ;{ }^{* *} p<0.01$.

Note. VFT $=$ Verbal Fluency Test [36]; TMT $=$ Trail Making Test [47]; MCST $=$ Modified Card Sorting Test [41]; SCW = Stroop Colour Word Test [53]; GNT = Goal Neglect Test [14]; BADS = Behavioural Assessment of Dysexecutive Syndrome [57].

Table 5

ANOVA results upon neuropsychological results for patients with preserved intelligence, using two between-subject factors: condition (schizophrenic patients versus control subjects) and nationality (Japanese versus British)

\begin{tabular}{|c|c|c|c|c|c|c|c|}
\hline & & \multicolumn{2}{|c|}{ Condition } & \multicolumn{2}{|c|}{ Nationality } & \multicolumn{2}{|c|}{ Condition $\times$ Nationality } \\
\hline & & $F$ & $\mathrm{P}$ & $F$ & $P$ & $F$ & $\mathrm{P}$ \\
\hline VFT & & 0.724 & 0.399 & 0.599 & 0.443 & 2.496 & 0.121 \\
\hline TMT B- $A$ & & 50.224 & $<0.001^{* *}$ & 10.884 & $0.002 * *$ & 2.294 & 0.137 \\
\hline MCST & & 21.894 & $<0.001^{* *}$ & 0.043 & 0.836 & 0.032 & 0.858 \\
\hline SCW & & 27.762 & $<0.001^{* *}$ & 1.043 & 0.313 & 1.642 & 0.207 \\
\hline GNT & & 24.587 & $<0.001^{* *}$ & 1.089 & 0.303 & 0.434 & 0.514 \\
\hline \multirow[t]{7}{*}{ BADS } & Rule shift cards & 7.981 & $0.007^{* *}$ & 0.156 & 0.694 & 0.020 & 0.888 \\
\hline & Action programme & 5.718 & $0.021^{*}$ & 2.390 & 0.129 & 0.949 & 0.335 \\
\hline & Key search & 8.234 & $0.006^{* *}$ & 5.087 & $0.029 *$ & 0.409 & 0.526 \\
\hline & Temporal judgement & 4.032 & $0.049^{*}$ & 9.243 & $0.004^{* *}$ & 0.101 & 0.752 \\
\hline & Zoo map & 29.586 & $<0.001^{* *}$ & 0.123 & 0.727 & 0.145 & 0.706 \\
\hline & Modified six elements & 7.988 & $0.007^{* *}$ & 0.489 & 0.488 & 0.489 & 0.488 \\
\hline & Profile score & 23.578 & $<0.001$ & 2.269 & 0.139 & 0.001 & 0.981 \\
\hline
\end{tabular}

${ }^{*} p<0.05 ;{ }^{* *} p<0.01$.

Note. VFT $=$ Verbal Fluency Test [36]; TMT $=$ Trail Making Test [47]; MCST $=$ Modified Card Sorting Test [41]; SCW $=$ Stroop Colour Word Test [53]; GNT = Goal Neglect Test [14]; BADS = Behavioural Assessment of Dysexecutive Syndrome [57].

In view of the potential influence of pharmacotherapy $[12,34,36]$, the effect of drugs on neuropsychological performance was analysed. In fact, 14 out of 22 British patients were taking clozapine among other drugs, and 12 this medication alone, whereas the Japanese patients were taking haloperidol, levomepromazine, and chlorpromazine (clozapine is not yet authorised in Japan). Twelve British schizophrenic patients on clozapine monotherapy were compared with 10 Japanese patients on typical neuroleptics equalised for the averages of age, duration of education, duration of illness, and current intelligence indicated by RAPM score. ANCOVA, using the RAPM score as covariate, did not reveal significant differences between the
British clozapine group and the Japanese traditional neuroleptics group on any of the executive tests.

\subsection{Summary of results}

EFs in the Japanese sample were as impaired as in the British one. Irrespective of nationality, the deficits in EFs, as assessed by most executive tests, were more severe than the deficits in RAPM-based intelligence. In both clinical samples, there was a severe impairment on formal executive tests, such as the TMT, the MCST, the SCW, and the GNT, although performance on the VFT was not significantly impaired. These groups also performed poorly on everyday executive tests. Impairment 
was apparent on some sub-tests and the profile score of the BADS in the two schizophrenic groups. The same profile of impairment was found amongst subjects of both cultures with minimal intellectual deterioration. Type of neuroleptics did not seem to influence the profile of impairment very much.

\section{Discussion}

\subsection{Relationship of EFs to intelligence}

These findings suggest that both intelligence and EFs, including everyday executive abilities, were affected regardless of culture. The $\mathrm{z}$ score profiles of the four groups on the neurobehavioural measures are plotted on Fig. 1. The mean of the British control group is represented by the zero line, with $S D=1$ for all functions. As Fig. 1 shows, there was a clear difference in performance pattern between the clinical groups and the healthy comparison groups. Both clinical groups made poor performance on four of the five executive tests, and this seemed to be not necessarily related to the level of intelligence of either group. For example, in the two schizophrenic groups, differences between the RAPM on the one hand and the TMT, the MCST and the BADS on the other were more than two standard deviations away from the British control subjects, and between the RAPM and the SCW there was more than one standard deviation. Contrary to expectation, both clinical groups performed less poorly on the VFT than on the RAPM intelligence test. The same neuropsychological pattern was shared by both Japanese and British schizophrenic patients.

RAPM-based intelligence in the two schizophrenic groups was significantly lower than that in the two control groups. The fact that the measurements of the British schizophrenic group were lower than those of the British control group in terms of the RAPMbased intelligence (post-hoc Tukey: $p=0.002$ ) in spite of matched NART-based premorbid intellectual level meant the intellectual decline in the former group which came about as a result of the onset of schizophrenia. Due to the absence of a test analogous to the NART in the Japanese language, we could not exactly estimate premorbid intelligence in the Japanese patients. For this reason, the difference between the Japanese schizophrenic and control groups in terms of the RAPM performance (post-hoc Tukey: $p=0.001$ ) did not necessarily signify the intellectual deterioration in the Japanese schizophrenic patients.
The ANCOVA result suggested that it seemed to be difficult to attribute executive deficits merely to that of lowered levels of intelligence. It is unfortunate that we cannot completely rule out the possibility that, beyond a certain level of decline, relatively small intellectual decrements might be causally associated with larger deficits in EFs in some non-linear fashion. Indeed, in order to cope with this problem to some extent, we analysed data from the minimal intellectual decline subgroups. But, we grant that the hypothesis of psychometric independence of EFs from general intelligence should be tested on the basis of a much larger sample of schizophrenic patients whose current intelligence is matched with that of the control subjects.

At least, we may exclude the possibility that the findings are attributed merely to the limited sensitivity of the RAPM. Due to its relationship to 'fluid intelligence', this test was chosen in preference to the Wechsler Adult Intelligence Scale (WAIS) [55]. The RAPM [46] consists of visual pattern matching and analogy problems, pictured in abstract designs which require the subject to perceive and discriminate spatial, design, and numerical relationships. Like Cattell's Culture Fair [7], this test is intended to be a 'culturefree' test of general ability in that it requires neither language nor academic skills for success but, rather, reasoning and novel problem solving for which familiar solutions are not available [30]. In this respect, the RAPM shares the characteristics of 'fluid intelligence' tests with Cattell's Culture Fair.

Although we did not actually administer the WAIS to the patients, it is unlikely that schizophrenic patients in our sample would potentially have made a poor performance on the WAIS. This is in the light of the fact that the schizophrenic groups in our sample showed relatively good performance on the VFT. Interestingly enough, this fact contrasts sharply to the study of Crawford et al. [10] in which patients with schizophrenia were associated with significantly lower VFT scores than would be predicted on the basis of Verbal IQ scores of the WAIS. Although, at first glance, the present findings of relatively well-preserved VFT performance contradict the observation of Crawford et al., there may be two implications; (1) the verbal IQ of schizophrenic patients should have been, by no means, worse than that of the control subjects in the present study and (2) the patients might be associated with larger deficits in a domain of EFs that are not explained by the VFT but are nevertheless assessed by other executive tests such as the TMT, the MCST, the SCW and the BADS. Be that as it may, these results can be cautiously interpreted 


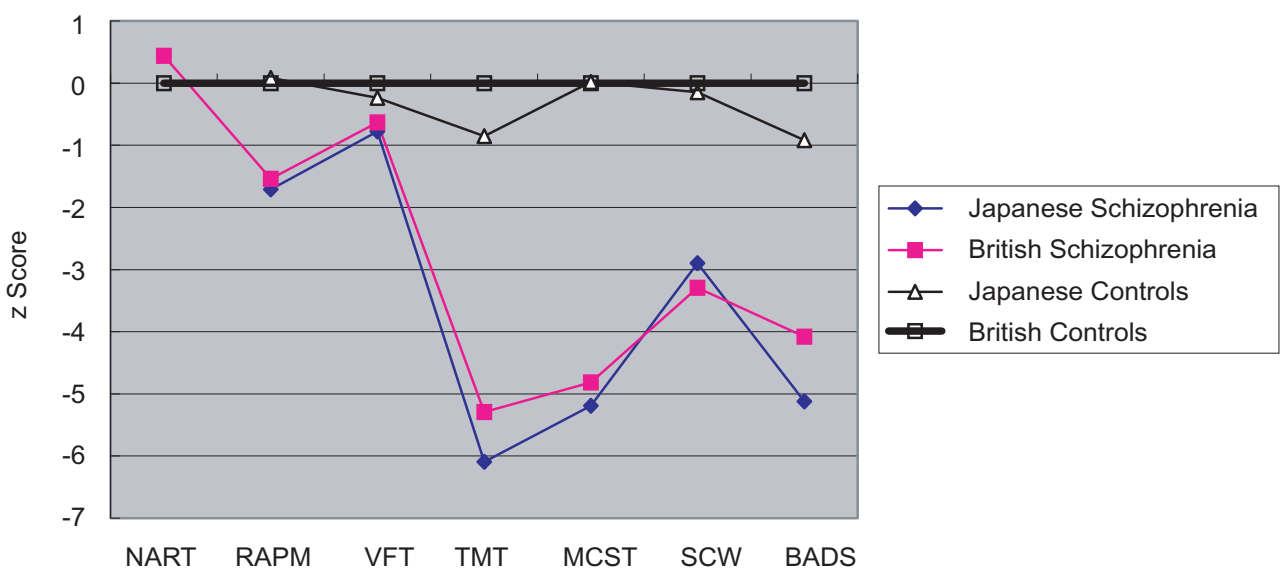

Fig. 1. The profiles of impairment: the Japanese and British patients, the Japanese and British control subjects (standard deviations from mean of the British control group). Note. NART = National Adult Reading Test [42]; RAPM = Raven Advanced Progressive Matrices [46]; VFT = Verbal Fluency Test [36]; TMT = Trail Making Test [47]; MCST = Modified Card Sorting Test [41]; SCW = Stroop Colour Word Test [53]; BADS = Behavioural Assessment of Dysexecutive Syndrome [57].

as suggesting that poor performance on executive tests was not entirely due to the impairment of intelligence relating to all cognitive domains.

This interpretation of the data in terms of the relationship between EFs and intellectual impairment in schizophrenia is consistent with the following findings. Applying the neuropsychological case-study approach to five chronic schizophrenic subjects, Shallice et al. [51] revealed ubiquitous impairment in performance on tests sensitive to frontal executive deficits. Using the BADS, Evans et al. [17] reported that significant impairment continued to be found in patients with chronic schizophrenia even after those with remarkable decline in general intellectual functioning had been excluded. Elliott et al. [15] compared schizophrenic and control subjects on a computerised test of attentional set-shifting. Chronic schizophrenic patients were found to experience significant difficulty in shifting to a novel dimension and ignoring the previously relevant one, and this was equally true of a sub-sample of patients with preserved IQ.

These findings suggest that the disturbance of integration and organisation of behaviour seen in schizophrenia may be related to dysfunction in the frontal cortex and its allied structures. Although other localising hypotheses cannot be ruled out, they have less empirical support. A considerable body of evidence has accumulated in favour of a close association between frontal lobe damage and impaired performance on executive tests $[9,44,48]$.

\subsection{Cross-cultural similarities of schizophrenia}

Our study also shows that in spite of major cultural differences, Japanese and British schizophrenic patients do not differ much in regard to their dysexecutive profile. Indeed, we should admit that the thematic content of some positive symptoms may be conditioned by culture. As for symptomatology, all we can say is at best that no prominent difference was found between the two schizophrenic groups in terms of positive syndrome, negative syndrome, anergia, thought disturbance, hyperactivation, paranoid and belligerent tendency, and depression (Table 2). These findings support the view of WHO (1979) that a class of common core symptoms universally underlies schizophrenia. However, the thematic aspect of psychopathology could not be verified, as the Positive and Negative Syndrome Scale (PANSS) [25] is only concerned with the form and severity of symptoms. It is also true that social factors in the development of cognitive abilities must be allowed for. Even so, it would seem that the basic neuropsychological disturbance of schizophrenia affects cognitive domains less influenced by cultural variation.

However, the finding that there is no statistically significant difference between the Japanese and the British samples, does not guarantee that no difference would ever be found if a larger sample or other instruments were to be used. Even so, the issue of the power of statistical analysis should not be allowed to obscure the main thrust of our study for the following reason. The purpose of this study was to specify the 'dysexec- 
utive syndrome' in Japanese schizophrenic patients as a stable 'syndrome'. Here, the term syndrome means a unique constellation of signs and symptoms which occur together frequently enough to suggest an underlying disease process [54]. Its central connotation is that the signs and symptoms occur with each other much more often than each of them arising separately, together with other signs and symptoms. Our current interest, therefore, lies in the simultaneous appearance of a constellation of neuropsychological findings specific to DES in Japanese as well as British patients. Thus, we emphasise the concurrence of lowered scores on a number of executive tests, with intelligence relatively unimpaired.

The finding of similar neuropsychological abnormalities in patients with schizophrenia from different cultures may possibly support the view that this condition has a biological basis and that it is present in various cultures. Such cultural ubiquity is also observed in anti-saccadic eye movement [1] and N400 event-related potential [5]. The cross-cultural stability of neurobiological data obtained by different methods contrasts markedly with the finding that socio-cultural variables are significant mediators of cross-ethnic symptom differences [6]. The above evidence refutes the extreme cultural-relativistic view of schizophrenia and supports Murphy's [38] view: “(r)ather than being simply violations of the social norms of particular groups, as labelling theory suggests, symptoms of mental illness are manifestations of a type of affliction shared by virtually all mankind."

\subsection{Cross-cultural applicability of the 'ecologically valid' test}

The ecological validity of the BADS, which was devised to measure everyday executive problems [57], does not seem to interfere with its cross-cultural applicability very much. The requirements of each subtask are similar to those of real-life activity, and, consequently, reflect the daily demands of the culture in which the BADS was devised. Still, no significant difference was found in test performances between Japanese and British schizophrenic patients, suggesting that the BADS can be applied equally to Japanese subjects, and, perhaps even individuals of other nationalities.

\subsection{Application of neuropsychological assessment to clinical practice in a multi-ethnic society}

Because the neuropsychological assessment of our samples was resistant to socio-cultural variables, it can be deduced that neuropsychological assessment in general should be considered useful in the severity assessment, outcome prediction and the management and rehabilitation of patients with schizophrenia in a multiethnic milieu.

Clinical decision is at present still determined by descriptive information provided by subjects with schizophrenia and by their care-givers [2,59]. The central weakness of this approach is its susceptibility to socio-cultural factors [29].

In this respect, neuropsychological assessment can supplement subjective-phenomenological data. While we found a quantitative concordance between Japanese and British patients on a wide range of frontal executive tests, we also observed common examination behaviour in schizophrenic patients with DES, irrespective of nationality. When qualitative aspects of aberrant behaviour as well as numerical evidence are appropriately analysed, psychometric instruments may provide added clinical information relevant to the understanding and management of schizophrenic patients.

\section{Conclusion}

This study revealed that both British and Japanese groups of schizophrenic patients showed equally severe impairment in the BADS as well as conventional executive tests (although verbal fluency and intelligence were less impaired). It is unlikely that poor performance on executive tests was due entirely to the impairment of intelligence relating to all cognitive domains. This is because similar neuropsychological deficits were also found in patients with minimal intellectual deterioration, irrespective of cultural origin. These findings suggested that socio-cultural background does not affect the dysexecutive profile of schizophrenia very much, and that neuropsychological assessment may possibly provide added clinical information relevant to the management and rehabilitation of schizophrenic patients across different cultures.

\section{Acknowledgements}

We thank all staff members of the Cambridge Psychiatric Rehabilitation Service, Muroi Hospital, Oyama 
Fujimidai Hospital, and Nasu Hillside Hospital who allowed us to examine patients under their care. We are also grateful to Professors Eric Chen for comments upon earlier drafts of this paper. We have received no external funding for this research.

\section{References}

[1] J.S. Allen, A.J. Lambert, F.Y. Johnson, K. Schmidt and K.L. Nero, Antisaccadic eye movements and attentional asymmetry in schizophrenia in three Pacific populations, Acta Psychiatrica Scandinavica 94 (1996), 258-265.

[2] American Psychiatric Association, Diagnostic and Statistical Manual of Mental Disorders: Fourth Edition, American Psychiatric Association, Washington, DC, 1994.

[3] A.D. Baddeley, Working Memory, Oxford University Press, Oxford, 1986.

[4] F.M. Benes, I. Sorensen, S.L. Vincent, E.D. Bird and M. Sathi, Increased density of glutamate-immunoreactive vertical processes in superficial laminae in cingulate cortex of schizophrenic brain, Cortex 2 (1992), 503-512.

[5] M.A. Bobes, Z.X. Lei, S. Ibanez, H. Yi and M. Valdes-Sosa, Semantic matching of pictures in schizophrenia: a crosscultural ERP study, Biological Psychiatry 40 (1996), 189-202.

[6] J.S. Brekke and C. Barrio, Cross-ethnic symptom differences in schizophrenia: the influence of culture and minority status, Schizophrenia Bulletin 23 (1997), 305-316.

[7] R.B. Cattell and A.K.S. Cattell, Test of ' $g$ ': Culture Fair, Institute for Personality and Ability Testing, Champaign, IL, 1973.

[8] R. Chandrasena, Schneider's First Rank Symptoms: An international and interethnic comparative study, Acta Psychiatrica Scandinavica 76 (1987), 574-578.

[9] J.R. Crawford, ed., Assessment of Attention and Executive Functions, Neuropsychological Rehabilitation 8 (1998), Special Issue.

[10] J.R. Crawford, M.C. Obonswain and M. Bremner, Frontal lobe impairment in schizophrenia: relationship to intellectual functioning, Psychological Medicine 23 (1993), 787-790.

[11] J.M. Davis, Comparative dose and cost of antipsychotic medication, Archives of General Psychiatry 33 (1976), 858-861.

[12] A.Y. Deutch, Mechanisms of action of clozapine in the treatment of neuroloptic-resistant and neuroleptic-intolerant schizophrenia, European Psychiatry 10 (1995), 39-46.

[13] J. Duncan, Attention, intelligence, and the frontal lobes, in: The Cognitive Neurosciences, M.S. Gazzaniga, ed., MIT Press, Cambridge, MA, 1995, pp. 721-733.

[14] J. Duncan, H. Emslie, P. Williams, R. Johnson and C. Freer, Intelligence and the frontal lobe: The organization of Goaldirected behavior, Cognitive Psychology 30 (1996), 257-303.

[15] R. Elliott, P.J. McKenna, T.W. Robbins and B.J. Sahakian, Neuropsychological evidence for frontostriatal dysfunction in schizophrenia, Psychological Medicine 25 (1995), 619-630.

[16] J.B. Enright and W.R. Jaeckle, Psychiatric symptoms and diagnosis in two subcultures, International Journal of Social Psychiatry 9 (1963), 12-17.

[17] J.J. Evans, S.E. Chua, P.J. McKenna and B.A. Wilson, Assessment of the Dysexecutive syndrome in schizophrenia, Psychological Medicine 27 (1997), 635-646.

[18] C.D. Frith, The Cognitive Neuropsychology of Schizophrenia, Lawrence Erlbaum Associates, Hove, 1992.
[19] E. Goldberg, Akinesia, tardive dysmentia, and frontal lobe disorders in schizophrenia, Schizophrenia Bulletin 11 (1995), 255-263.

[20] T.E. Goldberg, J.R. Kelsoe, D.R. Weinberger, N.H. Pliskin, P.D. Kirwin and K.F. Berman, Performance of schizophrenic patients on putative neuropsychological tests of frontal lobe function, International Journal of Neuroscience 42 (1988), 51-58.

[21] R.S. Goldman, B.N. Axelrod, R. Tandon and S. Bernet, Analysis of executive functioning in schizophrenics using the Wisconsin Card Sorting Test, Journal of Nervous and Mental Diseases 179 (1991), 507-508.

[22] T. Hatta and S.J. Dimond, Comparison of lateral differences for digit and random-form recognition in Japanese and Westerners, Journal of Experimental Psychology, Human Perceptual Performance 6 (1980), 174-368.

[23] A.R. Jensen, Bias in Mental Testing, Methuen \& Co., London, 1980.

[24] M.M. Katz, K.O. Sanborn, H.A. Lowery and J. Ching, Ethnic studies in Hawaii: Psychopathology and social deviance, in: The Nature of Schizophrenia, L. Wynne, R.L. Cromwell and S. Matthysse, eds, John Wiley \& Sons, Inc., New York, 1978, pp. 572-584.

[25] S.R. Kay, A. Opler and A. Fiszbein, Positive and Negative Syndrome Scale (PANSS) Manua, Multi-Health Systems Inc., Toronto, 1990.

[26] D.J. King, Neuroleptics and the treatment of schizophrenia, in: Seminars in Clinical Psychopharmacology, D.J. King, ed., Gaskell, London, 1995, pp. 259-327.

[27] B. Kolb and I.Q. Whishaw, Performance of schizophrenic patients on tests sensitive to left or right frontal, temporal or parietal function in neurological patients, Journal of Nervous and Mental Disease 171 (1983), 435-443.

[28] E. Kraepelin, Vergleichende Psychiatrie, Zentralblatt fir Nervenheilkunde und Psychiatrie 15 (1904), 433-437.

[29] J. Leff, International variations in the diagnosis of psychiatric illness, British Journal of Psychiatry 131 (1977), 329-338.

[30] M.D. Lezak, Neuropsychological Assessment: Third Edition, Oxford University Press, Oxford, 1995.

[31] P.F. Liddle and D.L. Morris, Schizophrenic symptoms and frontal lobe performance, British Journal of Psychiatry 158 (1991), 340-345.

[32] K.M. Lin and A.M. Kleinman, Psychopathology and clinical course of schizophrenia: A cross-cultural perspective, Schizophrenia Bulletin 14 (1988), 555-567.

[33] R.E. Litman, D.W. Hommer, T. Clem, M.L. Ornsteen, C. Ollo and D. Pickar, Correlation of Wisconsin Card Sorting Test performance with eye tracking in schizophrenia, American Journal of Psychiatry 148 (1991), 1580-1582.

[34] H.Y. Meltzer, Dimensions of outcome with clozapine, British Journal of Psychiatry 160 (1992), 46-53.

[35] E. Miller, Verbal fluency as a function of a measure of verbal intelligence and in relation to different types of cerebral pathology, British Journal of Clinical Psychology 23 (1984), 53-57.

[36] A.M. Mortimer, Cognitive function in schizophrenia: Do neuroleptics make a difference? Pharmacology, Biochemistry and Behavior 56 (1997), 789-795.

[37] E.M. Moss, R.J. Davidson and C. Saron, Cross-cultural differences in hemisphericity: EEG asymmetry discriminates between Japanese and Westerners, Neuropsychologia 23 (1985), 131-135.

[38] J.M. Murphy, Psychiatric labeling in cross-cultural perspective, Science 191 (1976), 1019-1028. 
[39] H.B.N. Murphy, Culture and schizophrenia, in: Culture and Psychopathology, I. Al-Issa, ed., University Park Press, Baltimore, 1982, pp. 221-249.

[40] H.B.M. Murphy, E.D. Wittkower, J. Fried and H. Ellenberger, A cross-cultural survey of schizophrenic symptomatology, International Journal of Social Psychiatry 9 (1963), 237-249.

[41] H.E. Nelson, A modified card sorting task sensitive to frontal lobe defects, Cortex 12 (1976), 313-324.

[42] H. Nelson and J.R. Willison, The National Adult Reading Test (NART), (2nd ed.), NFER-Nelson, Windsor, 1991

[43] C. Pantelis, T.R.E. Barnes and H.E. Nelson, Is the concept of frontal-subcortical dementia relevant to schizophrenia? British Journal of Psychiatry 160 (1992), 442-460.

[44] P. Rabbitt, ed., Methodology of Frontal and Executive Function, Psychology Press, Hove, 1997.

[45] J.C. Raven, Advanced Progressive Matrices: Set I, Oxford Psychologists Press, Oxford, 1962.

[46] J.C. Raven, Advanced Progressive Matrices, Oxford Psychologists Press, Oxford, 1976.

[47] R.M. Reitan, Validity of the trail-making test as an indication of organic brain damage, Perceptual and Motor Skills $\mathbf{8}$ (1958), 271-276.

[48] A.C. Roberts, T.W. Robbins and L. Weiskrantz, eds, The Prefrontal Cortex: Executive and Cognitive Functions, Oxford University Press, Oxford, 1998.

[49] C. Schooler and W. Caudill, Symptomatology in Japanese and American schizophrenics, Ethnolog 3 (1964), 172-178.

[50] J. Schröder, F. Wenz, L.R. Schad, K. Baudendistel and M.V. Knopp, Sensorimotor cortex and supplementary motor area changes in schizophrenia: a study with functional magnetic resonance imaging, British Journal of Psychiatry 167 (1995), 197-201.
[51] T. Shallice, P.W. Burgess and C.D. Frith, Can the neuropsychological case-study approach be applied to schizophrenia? Psychological Medicine 21 (1991), 661-673.

[52] D. Tamlyn, P.J. McKenna, A.M. Mortimer, C.E. Lund, S. Hammond and A.D. Baddeley, Memory impairment in schizophrenia: its extent, affiliations and neuropsychological character, Psychological Medicine 22 (1992), 101-115.

[53] M.R. Trenerry, B. Crosson, J. DeBoe and W.R. Leber, Stroop Neuropsychological Screening Test Manual, Psychological Assessment Resources, Odessa, 1989.

[54] K.W. Walsh, Understanding Brain Damage. A Primer of Neuropsychological Evaluation. Second Edition, Churchill Livingstone, Inc., London, 1991.

[55] D. Wechsler, Wechsler Memory Scale-Revised Manual, The Psychological Cooperation, San Antonil Tx, 1987.

[56] D.R. Weinberger, K.F. Berman and B.P. Illowsky, Physiological dysfunction of dorsolateral prefrontal cortex in schizophrenia. III. A new cohort and evidence for a nomoanimergic mechanism, Archives of General Psychiatry 45 (1988), 609-615.

[57] B.A. Wilson, N. Alderman, P. Burgess, H. Emslie and J.J. Evans, Behavioural Assessment of the Dysexecutive Syndrome $(B A D S)$, Thames Valley Test Company, Bury St. Edmunds, 1996.

[58] World Health Organization, Schizophrenia: An International Follow-up Study, Wiley, New York, 1979.

[59] World Health Organization, The ICD-10 Classification of Mental and Behavioural Disorders: Clinical Descriptions and Diagnostic Guideline, World Health Organization, Geneva, 1992. 


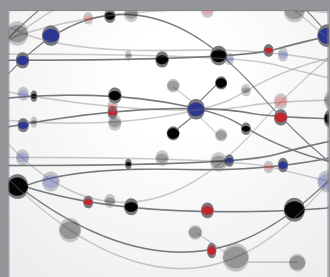

The Scientific World Journal
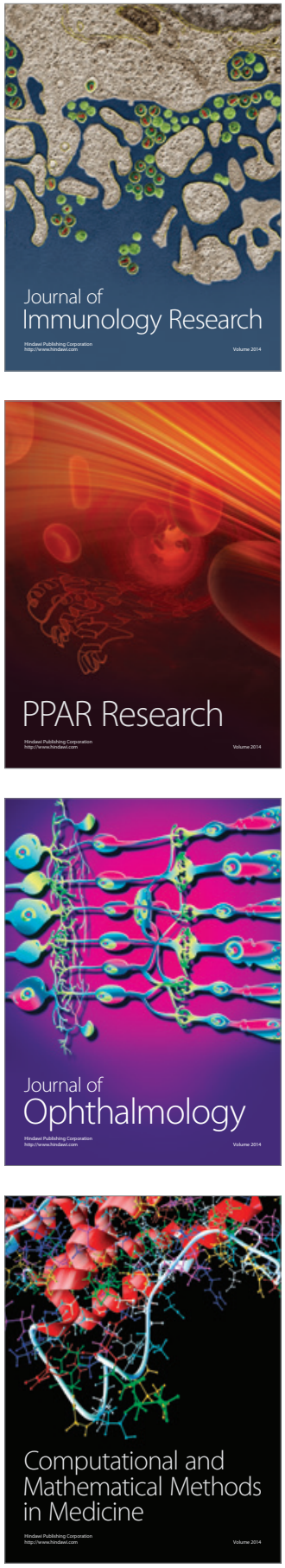

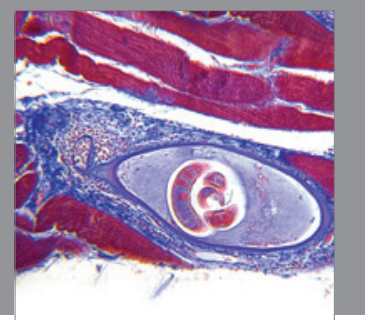

Gastroenterology

Research and Practice
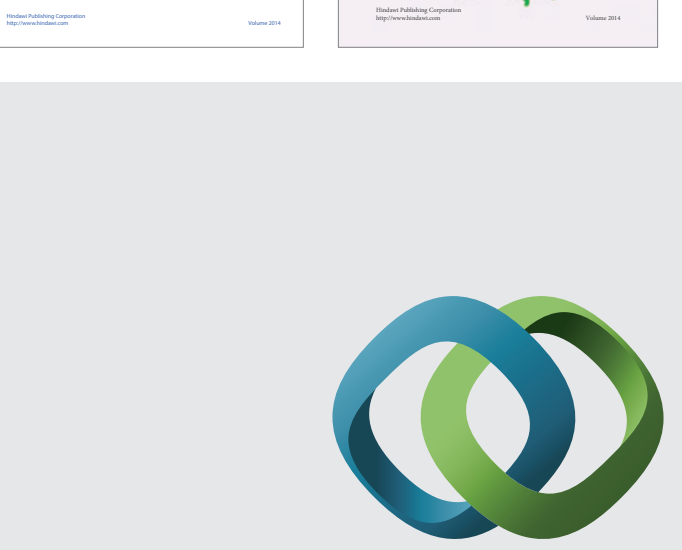

\section{Hindawi}

Submit your manuscripts at

http://www.hindawi.com
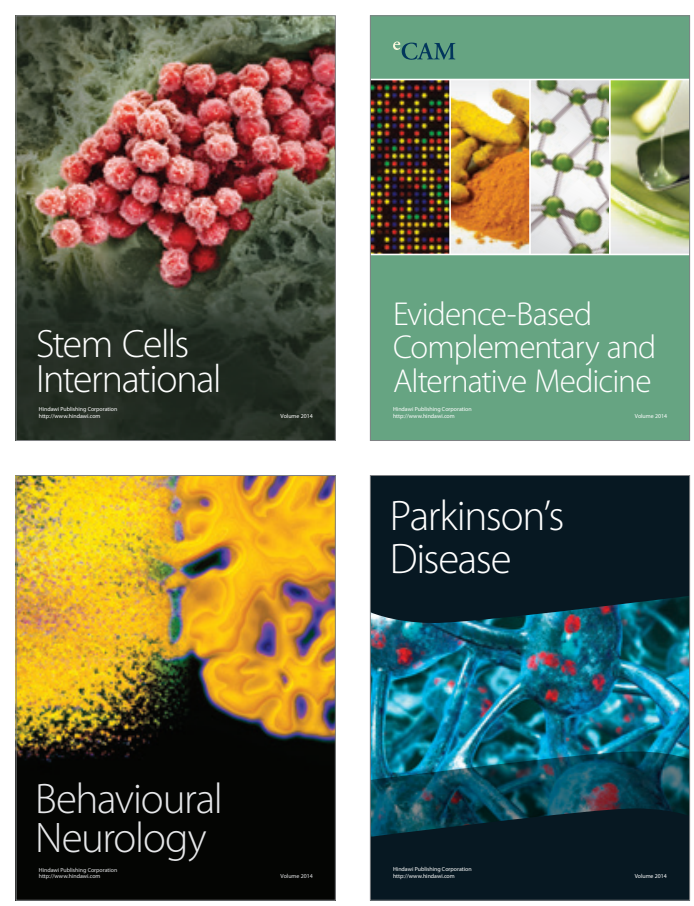

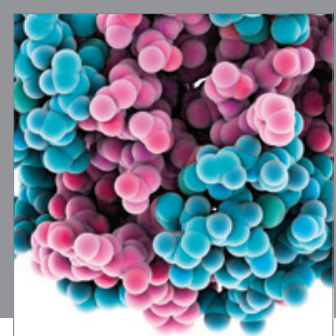

Journal of
Diabetes Research

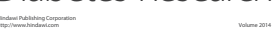

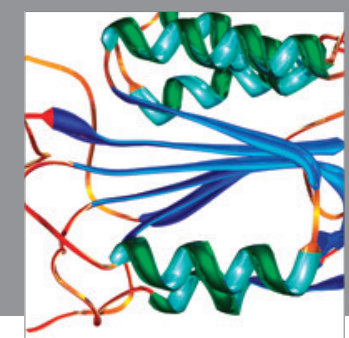

Disease Markers
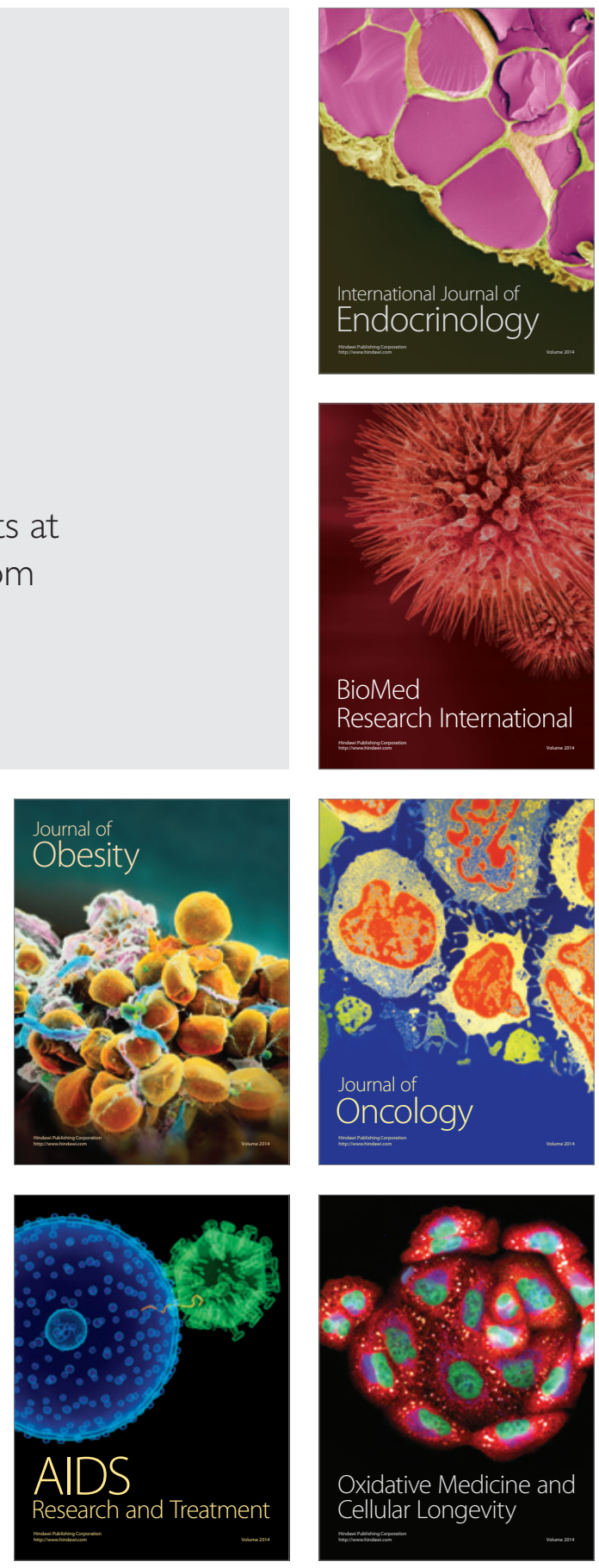